\title{
The MIND method: A decision support for optimization of industrial energy systems - Principles and case studies
}

Magnus Karlsson

The self-archived version of this journal article is available at Linköping University Electronic Press:

http:/ / urn.kb.se/ resolve?urn=urn:nbn:se:liu:diva-62633

N.B.: When citing this work, cite the original publication.

Karlsson, M., (2011), The MIND method: A decision support for optimization of industrial energy systems - Principles and case studies, Applied Energy, 88(3), 577-589.

https:// dx.doi.org/ 10.1016/j.apenergy.2010.08.021

Original publication available at:

https:/ / dx.doi.org/ 10.1016/j.apenergy.2010.08.021

Copyright: Elsevier

http:// www.elsevier.com/

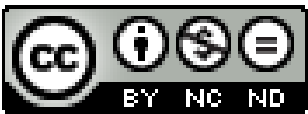




\title{
The MIND method: a decision support for optimization of industrial energy systems - principles and case studies
}

\author{
Magnus Karlsson ${ }^{*}$ \\ Department of Management and Engineering, Division of Energy Systems, \\ Linköping University, SE-581 83 Linköping, Sweden
}

\begin{abstract}
Changes in complex industrial energy systems require adequate tools to be evaluated satisfactorily. The MIND method (Method for analysis of INDustrial energy systems) is a flexible method constructed as decision support for different types of analyses of industrial energy systems. It is based on Mixed Integer Linear Programming (MILP) and developed at Linköping University in Sweden. Several industries, ranging from the food industry to the pulp and paper industry, have hitherto been modelled and analyzed using the MIND method. In this paper the principles regarding the use of the method and the creation of constraints of the modelled system are presented. Two case studies are also included, a dairy and a pulp and paper mill, that focus some measures that can be evaluated using the MIND method, e.g. load shaping, fuel conversion and introduction of energy efficiency measures. The case studies illustrate the use of the method and its strengths and weaknesses. The results from the case studies are related to the main issues stated by the European Commission, such as reduction of greenhouse gas emissions, improvements regarding security of supply and increased use of renewable energy, and show great potential as regards both cost reductions and possible load shifting.
\end{abstract}

Keywords: Decision support systems, Mixed Integer Linear Programming, Energy efficiency, Industrial energy systems, Load shaping

\footnotetext{
* Corresponding author. Tel.: +46-13-285739; fax: +46-13-281788. E-mail address: magnus.karlsson@liu.se.
} 


\section{Introduction}

The focus on energy efficient systems is becoming more and more important in the European Union. The European Commission focuses on three major issues in relation to energy efficiency: (1) to improve the competitiveness of European companies, since numerous studies claim that the European Union could save at least $20 \%$ of its present energy use in a cost-effective manner; (2) to reduce the impact of global warming, as it is stated that energy saving is the quickest, most cost-effective way of reducing greenhouse gas emissions; and (3) to improve security of supply in the union, since it is stated that by 2030 , on the basis of present trends, the EU will be $90 \%$ dependent on imports for its oil needs and $80 \%$ dependent as regards gas [1]. In 2008, the "20-20-20 objectives" were launched, representing commitments stated by the EU for Europe including (1) a reduction of greenhouse gas emissions by $20 \%$ by 2020 , compared to 1990 levels, (2) an increase in the level of renewable energy in the EU's final energy mix from $8.5 \%$ in 2005 to $20 \%$ by 2020 , and (3) the noncompulsory target of reducing its global primary energy use by $20 \%$ by 2020 [2].

Implementation of measures increasing energy efficiency in industrial companies are thus desirable and the importance of having a comprehensive view of the systems when making such changes in industry is emphasized in several studies, e.g. [3,4]. This is crucial, as industrial systems form complex relations both within the industrial equipment unit at plant level and in the interaction with their surroundings. The development of computer-based decision support increases the possibility to make as adequate analyses as required of different complex systems $[3,5]$.

Simulation is one well-established technique for analyzing different systems but there are others, such as the Monte Carlo method and neural networks, e.g. [6,7,8]. Another method is mathematical programming, which is a powerful tool when mathematical relations can be identified in the analyzed system $[9,10]$. Such values are often available when analyzing industrial systems (extracted from monitoring systems or through measurements) and one subgroup - mixed integer linear programming (MILP) - is, according to Grossmann and Santibanez [11], especially suitable for such problems. An extensive review regarding the use of mathematical programming in industries is presented in an article by Grossman et al. [12], and mathematical programming has since then been used extensively and recent industrial examples where MILP is used are found in e.g. [13,14]. 
The decision support software reMIND, based on the MIND method, is a model framework constructed to be able to model different types of industries under different specific conditions. MIND is an abbreviation of 'Method for analysis of INDustrial energy systems' and the method is based on MILP. The MIND method has been used as decision support in several industries, e.g. the steel industry and the automobile manufacturing industry. Different aspects have been elucidated with the method, such as the best operational and investment strategies, with the possibility to consider both economic and environmental aspects $[15,16,17]$.

Two case studies are included in this paper: a dairy and a pulp and paper mill. In the two case studies, the modelling focuses-five important aspects: fuel conversion, energy efficiency measures, load shifting, storing and system boundary expansion. In the two different case studies, the MIND method is used in different ways and a discussion about the usefulness, based on these case studies, is accomplished in the paper when related to the main issues stated by the European Commission [1,2] as discussed above. The constraints that can be included in the MIND method are also described in the paper and the need for different kinds of approaches when different kinds of systems with different time perspectives and different purposes, such as in the two case studies, are analyzed. A comparison and analysis of the two case study models are also made to analyze differences in, for example, the size of the problems.

\section{Mixed Integer Linear Programming}

Mixed Integer Linear Programming (MILP) is a well-established method for analyzing different systems $[9,10]$. A MILP problem includes an objective function, several variables, and constraints. The objective function comprises different variables that are minimized or maximized depending on the purpose. The constraints represent separate restrictions in the problem and limit the permissible solution set and, like the objective function, they consist of a number of variables. The integers included in a MILP problem can be used to consider a choice of any kind, but also artifacts that are to be integers, such as pumps and automobiles. Non-linear functions can be linearized piece-wise using integers and thus be included in a MILP problem. MILP problems are solved by using different kinds of mathematical 
algorithms, e.g. Cutting plane or Branch and bound [9]. A MILP problem is defined according to Equation 1 (objective function) and Equation 2 (constraints).

Objective:

$$
\min \sum_{v=1}^{V} \sum_{w=1}^{W}\left(c_{1, v} x_{v}+c_{2, w} y_{w}\right)
$$

Subject to:

$$
\sum_{v=1}^{V} \sum_{w=1}^{W}\left(c_{1, v, z} x_{v}+c_{2, w, z} y_{w}\right)\left\{\begin{array}{l}
\leq \\
= \\
\geq
\end{array} C_{z}, \forall z=1,2, \ldots, Z\right.
$$

\section{Characteristics of the MIND method and field of application}

\subsection{A brief description of the MIND method}

The MIND method was originally developed to model different types of industrial energy systems. Nonetheless, it is also possible to analyze and model systems of a more general character. However, applications of the method have so far, in principle, been limited to industries only and industries in cooperation with other players, such as district heating systems. The presentation in this chapter is based on the assumption that the method is used for modelling industrial energy systems.

The cornerstones that according to Nilsson [18] are required in order to analyze an industrial energy system, were implemented in a Fortran-based [19] program called MIND, see e.g. [18]. Later, a Java-based [20] program, reMIND, was developed based on previous research. The basic interface of the reMIND software is shown in Fig. 1.

According to [16] the analysis basically includes four steps. In the first step, the real system has to be delimited, simplifications introduced and processes identified. In the second step, the model is built. The structure of the modelled industrial energy system is represented as a network of branches and nodes. The branches represent flows of any kind and are designated specified types of resource of their own, such as electricity, steam and material. The nodes may represent a whole industry, a process line or a single piece of equipment. Each node may comprise numerous functions to describe the functionality of the depicted unit. Time is 
divided into time steps to consider, for example, hourly, weekly and annual changes in the modelled system. A new model is generated for each case studied, implying that the model can be formulated according to the special conditions at each industrial site. The model is represented mathematically as a matrix of equations (including the objective function and the constraints), based on the simplification and delimitation of the problem identified in the initial step, where the branches and the nodes are denominated systematically. In the third step, the matrix of equations, generated by reMIND in a standardized format, is optimized in an optimization solver to determine the structure of units and operational settings that offers the best possible conditions for the analyzed industry. Normally, CPLEX [21] is used in reMIND but other solvers may be used, e.g. Lp_solve [22]. Any type of resource may be minimized but the system cost is usually minimized based on net present value calculations. The system cost comprises all costs that are included in the calculation, for example investment costs and the cost of energy and raw materials. During the optimization, the modelled units compete with each other to satisfy the demand during the analyzed period at the lowest possible cost. In addition to cost-optimization, it is also possible to optimize problems that include multiple criteria, for example environmental issues. In the final step, the results from the optimization solver are presented in a text-file that is constructed to be used in software such as Microsoft Excel. The results are analyzed and the model is validated.

\subsection{Aspects elucidated with the MIND method}

The MIND method can be used to elucidate a number of questions, such as the optimal operation strategy, i.e. how to operate the analyzed industrial site as cost-effectively as possible under the prevailing conditions, e.g. [16]. Another alternative is to study whether the structure of the processes can be improved, e.g. [23]. Such an analysis compares the use of existing units with new alternatives included in the model. A third option is to use the method to investigate how different boundary conditions, such as changes in electricity and fuel prices, influence the system, e.g. [24]. The analysis perspectives outlined can also be combined, e.g. [25]. Almost 50 scientific journal and conference publications, in addition to several reports, where the MIND method has been used, have hitherto been published but giving a review of earlier studies is not the central topic in this paper. However, it may be noted that the method has shown great potential for use in combination with other analysis tools such as pinch technology [26], Fourier analysis [27], and exergy analysis [28]. By using 
two methods that approach the problem on two different system levels, the strengths of each can be highlighted and their weaknesses suppressed.

\section{The functions included in reMIND}

A nomenclature to define the variables, parameters and sub-scripts in the following functions and constraints can be found at the end of the paper.

\subsection{Formulation of the objective function of a MILP problem using reMIND}

The objective function, including the possibility to apply a multi-objective approach, is enabled with reMIND by the following function:

$$
\min \sum_{n=1}^{N} a_{n} b_{n} \sum_{t=1}^{T} \sum_{m=1}^{M}\left(c_{m, t, n} x_{m, t}\right) ; x \geq 0
$$

It is also possible to constrain the objective function, which allows methods such as the $\varepsilon$-constraint method and normal boundary interaction to be applied [29]:

$$
\sum_{t=1}^{T} \sum_{m=1}^{M} c_{m, t, n} x_{m, t}\left\{\begin{array}{l}
\leq \\
= \\
\geq
\end{array}\right\} C_{n}, \forall n
$$

$C_{n}$ is a constant that sets the constraint of each objective $n$ for the whole analyzed period. This global constraint function enables a constraint to be set on each objective, for example maximum total cost or maximum total emissions.

\subsection{Formulation of functions connecting flows}

Flows can be connected in three ways in reMIND:

FlowDependency - This function allows non-linear relations between flows of the same type of resource to be depicted, divided into flows coming into the node $(i)$ and flows going out of the node $(j)$. In this function, the relation can also be set between two different types of resource, also indexed $i$ and $j$. The constraints needed to describe this functionality are presented in Equations 5 to 7.

$$
\sum_{j=1}^{J} x_{t, j}-\sum_{i=1}^{I} c_{t, p, s l o p e} \cdot x_{t, i}-c_{t, p, s t e p} \cdot Y_{t, p}=0, \forall p, t
$$




$$
\sum_{p=1}^{P} Y_{t, p}=1, \forall t
$$

FlowEquation - This function does not include the possibility to depict non-linear relations. On the other hand, it is possible to connect any number of flows to each other regardless of the types of resources. Equation 8 presents the constraints needed, where $i$ represents flows entering the node and $j$ represents flows leaving the node.

$$
\sum_{j=1}^{J} c_{t, j} x_{t, j}-\sum_{i=1}^{I} c_{t, i} \cdot x_{t, i}\left\{\begin{array}{l}
\leq \\
= \\
\geq
\end{array}\right\} C_{t}, \forall t
$$

FlowRelation - When different flows going in or out of a node need to be related to each other, this function can set a percentage share between the flows. Equations 9 and 10 present the constraints needed for this functionality and $i$ may be exchanged for $j$ in the constraints.

$$
\sum_{i=1}^{I} x_{t, i}=F R_{t}, \forall t
$$

$$
x_{t, i}\left\{\begin{array}{l}
\leq \\
= \\
\geq
\end{array}\right\} d_{t, i} \cdot F R_{t}, \forall i, t
$$

A flow can be set with both a lowest and a highest percentage share of the total flow entering or leaving the node as well as be set as a free variable.

\subsection{Formulation of functions limiting flows}

There are two different ways to specify a boundary in reMIND that limits different flows:

Boundary - When a Boundary function is specified, Equation 11 is created. $i$ may be exchanged for $j$ in the constraints.

$$
c_{t, \min } \leq \sum_{i=1}^{I} x_{t, i} \leq c_{t, \max }, \forall t
$$


BoundaryTop - This function is appropriate when there is a need for, or a restriction of, a specific amount of a type of resource over the whole of the analyzed period, or a part of it, that can be delivered to a node at any time steps, $t$, included in the function. The time steps $t$ are optional. $i$ may be exchanged for $j$ in the constraints.

$$
\begin{aligned}
& \sum_{i=1}^{I} x_{0, i}-\sum^{t} \sum_{i=1}^{I} L_{t} x_{t, i}=0 \\
& c_{\min } \leq \sum_{i=1}^{I} x_{0, i} \leq c_{\max }
\end{aligned}
$$

\subsection{Formulation of storage functions}

In Heidari Tari and Söderström [30,31], basic constraints to represent storage functions are presented. In these articles, the storage presentation is divided into material storage and energy storage. The constraints below (Equation 14-18) have been modified to represent a general formulation of storage functions, which include the possibility to model both material and energy storage. Some additional features and constraints compared to the earlier formulations are also included.

$$
\sum_{i=1}^{I} \eta_{t, 1} L_{t} x_{t, i}-\sum_{j=1}^{J} \frac{L_{t} x_{t, j}}{\eta_{t, 2}}+\eta_{(t-1), 3} S E_{(t-1)}-S E_{t}=0, \forall t
$$

$S E_{0}$ is the initial storage volume, which is set to 0 as default and by default the final storage volume is set to the same as the initial volume, but this can be changed.

Each flow included in the StorageEquation function can have individual boundaries according to Equation 15. $i$ may be exchanged for $j$ in the constraint.

$$
c_{t, 1} \leq x_{t, i} \leq c_{t, 2}, \forall i, t
$$

The maximum and minimum amount of flow entering or leaving a node can also be aggregated according to Equation 16. $i$ may be exchanged for $j$ in the constraint.

$$
c_{t, 3} \leq \sum_{i=1}^{I} x_{t, i} \leq c_{t, 4}, \forall t
$$


The maximum and minimum storage capacity is set by the following constraint.

$c_{t, 5} \leq S E_{t} \leq c_{t, 6}, \forall t$

The maximum allowable number of time steps to store a subject can be set according to Equation 18.

$\sum_{i=1}^{I} \eta_{t, 1} L_{t} x_{t, i}-\sum_{j=1}^{J} \frac{L_{t} x_{t, j}}{\eta_{t, 2}}-\sum_{t=t+1}^{T=t+1+M S T_{t}} \sum_{j=1}^{J} \frac{L_{t} x_{t, j}}{\eta_{t, 2} \prod_{g=1}^{G} \eta_{(t+g-2), 3}} \leq 0, \forall t, G=1,2,3 \ldots \leq M S T_{t}$

For each time step that is added in the summation for time steps in Equation 18, $G$ is increased by one (starting at one). The maximum value of $G$ is $M S T_{t}$.

\subsection{Formulation of batch processes}

Batches in a model are handled using the following constraints (Equation 19-25).

$\sum_{i=1}^{I} L_{t} x_{t, i}-\sum_{j=1}^{J} L_{(t+B T)} x_{(t+B T), j}=0, \forall t$

The maximum and minimum batch size is set by the following constraint.

$c_{t, 1} \cdot Y_{t} \leq \sum_{i=1}^{I} L_{t} x_{t, i} \leq c_{t, 2} \cdot Y_{t}, \forall t$

There are two alternatives for modelling the batch processes:

- The batches can occur any time during the time period

$$
\sum_{t}^{t+B T-1} Y_{t} \leq 1, \forall t
$$

- The batches occur at predetermined times during the time period

$$
\sum_{t}^{t+B T-1} Y_{t}=1, \forall t
$$

$$
Y_{1}=1
$$

An adjusting time, i.e. the number of time steps the batch process needs for other purposes, e.g. cleaning, can also be included. The constraints needed depend on whether the batches are predetermined or not. If the batches can occur at any time during the time period the 
constraint created by Equation 24 is used to complement the constraint created by Equation 21.

$Y_{t}+Y_{(t+B T+q-1)} \leq 1, \forall t, q$

If the batches are predetermined, Equation 22 is changed according to Equation 25.

$$
\sum_{t}^{t+B T+Q-1} Y_{t}=1, \forall t
$$

\subsection{Formulation of choices based on integer variables}

The logical restrictions may, for example, represent a choice between two different processes that are exclusive. Equations 26 and 27 are used to represent logical restrictions.

$$
\sum_{i=1}^{I} c_{t, i} Y_{t, i}+\sum_{i=j}^{J} c_{t, j} Y_{t, j}\left\{\begin{array}{l}
\leq \\
= \\
\geq
\end{array}\right\} C_{t}, \forall t
$$

$c_{t, 1} Y_{t, i} \leq x_{t, i} \leq c_{t, 2} Y_{t, i}, \forall t, i$

\subsection{Formulation of investment cost function}

When investing in any kind of equipment, the size of the unit needs to be considered. The constraints generated with the function InvestmentCost extract the largest size of the equipment used in the model, found in any of the time steps, and assign an appropriate price for that size. The rate of return, economic lifetime and technical lifetime can be included in the function. The disposal residual values can also be entered as fixed values or percentages, where the latter is dependent on the size of the optimal investment. Information regarding the formulation of the investment cost function can be found in [32].

\subsection{Formulation of not predetermined functions}

In cases when it is not possible to build an accurate model, using the predetermined functions to construct the constraints needed, the FunctionEditor function may be used. Using the FunctionEditor it is possible to generate constraints of different kinds in a flexible way, containing internal float variables and/or integer variables for the specific function, with a 
possibility to connect the variables to different time steps. The constraints are formulated directly via an editable window.

\section{Cases studied}

Two case studies are included in this paper to show the use of the MIND method in general and to show how the issues stated by the European Commission [1,2] as discussed in the introduction may be considered by using the method. However, not all reMIND's features can be shown in these case studies; the multi-objective approach, for example, is not included. A dairy and a pulp and paper mill are modelled in order to show the use of reMIND in different industries. The case studies are also selected to show the use of reMIND for different analysis lengths, different focuses, and when different types of measures are included. The models are simplified compared to reality but characteristics essential for the purpose of the modelling are included. The models are based on input data originating from parts of an existing dairy and from an existing pulp and paper mill, compiled in cooperation with staff.

The system cost of the models in the case studies is minimized and only considers costs for energy. This means that there is no charge for raw material and no revenues from the products. Only process-related energy demands are included in the models; demands such as ventilation and comfort are not. Time is divided into time steps to represent changes in energy prices during the analyzed periods.

\subsection{Case study of a dairy}

\subsubsection{The model of the dairy}

A one-day production, divided into 24 time steps, each representing one hour, is included in the model. The structure of the model is shown in Fig. 1, representing all cases but one, where an additional node, representing an absorption cooling machine to produce ice water, is included.

The different processes, such as buffers, tanks etc, are represented by nodes, while the branches represent flows of any kind. In this model, the white branches represent a flow of milk products, the dark grey arrows steam flow, the light grey branches electricity flow and 
the black branches a flow of ice water. Ice water is produced using an electrically powered cooling machine. In Table 1, input data for processes included in the model is presented.

When standardizing yoghurt, skim milk and whole milk are mixed in a $2 / 5$ relation, while sour cream is a mix between skim milk and cream milk in the relation 1/4. Cream milk is standardized using around 3 parts of skim milk and 2 parts of cream. In total, $15.5 \mathrm{~m}^{3}$ of yoghurt and $10.3 \mathrm{~m}^{3}$ of sour cream are to be produced over the course of one day.

\subsubsection{Analyzed cases in the dairy} In the dairy, six different cases are analyzed to show the influence of load shifting (Cases D2 and D3), fuel conversion (Case D4) and energy efficiency measures (Cases D5 and D6). Case D1 is a base case showing the present situation. A summary of the different cases is shown in Table 2 and the energy prices and fees included in the different cases are shown in Table 3 (the variable price of electricity is shown in Table 4).

\subsection{Case study of a pulp and paper mill}

\subsubsection{The model of the pulp and paper mill}

The aim of the company is to produce $650,000 t_{90}$ cardboard annually divided over two cardboard machines, CM7 and CM8. Of the total production, CM7 accounts for $40 \%$ and CM8 for $60 \%$. Production is assumed to be constant all year round but due to revisions and other operational breakdowns the operation time amounts to 8,400 hours annually, which is the figure used in the modelling. The model covers one year of production divided into 12 time steps, each representing one month. Chemi-thermomechanical pulp (CTMP) and sulphate pulp, together with purchased pulp (pulp bale), is delivered to the cardboard machines to sustain production. Each cardboard machine can produce more than one cardboard quality product, but to simplify the case study this has been limited and it is assumed that each cardboard machine can produce only one product quality.

The structure of the model is shown in Fig. 2, representing all cases (the nodes for the storages, the turbine + generator, and the district heating system are included in the model for the specific cases, described in 5.2.2). The different processes are, as for the dairy, represented in reMIND by nodes, such as the cardboard machines and boilers, while the 
branches represent flows of any kind. In this model the white branches represent flows of wood/pulp/paper products, the dark grey arrows steam flow, the light grey branches electricity flow and the black branches a flow of fuel. The input data for the processes included in the model are presented in Table 5 and the input data for the boilers in Table 6 . The prices used in the model are shown in Table 7.

\subsubsection{Analyzed cases in the pulp and paper mill}

In the pulp and paper mill four different cases are analyzed, as shown in Table 8, where Case $\mathrm{P} 1$ is the base case showing the present situation. Case P2 is a case showing the effect of storing oil and bark. The oil and bark storages are filled at the beginning of the analysis period and have to be filled up again at the end of the period and the total maximum volume is $320 \mathrm{GWh}$ for each storage. Oil can be stored during the whole year while bark can be stored for a maximum of 6 months. Case P3 represents a case with a possibility to produce electricity on-site. The maximum electricity production for the turbine amounts to $75 \mathrm{MW}$ and the turbine is assumed to have an "electricity efficiency" of 15\%, according to Equation 28 and a "total efficiency" of $95 \%$, according to Equation 29 .

$$
\text { Electricityefficincy }=\frac{\text { electricityproduction }}{\text { admissionsteamflow }}
$$

"Totalefficiency" $=\frac{\text { electricityproduction }+ \text { backpressuresteamflow }}{\text { admissionsteamflow }}$

The last case, Case P4, is included to show the effect of a change in the system boundary, by widening the system boundary with possibilities to deliver heat to a district heating system, with a total annual demand of $575 \mathrm{GWh}$. Information regarding the district heating system can be found in Table 7. To be able to make comparisons of the system cost an alternative district heating system is also modelled, called Case DH. Case DH includes an oil boiler, oil storage and a district heating system where the oil price, the characteristics of the oil boiler and the district heating demand are the same as shown in Table 6 and Table 7. The oil storage in Case $\mathrm{DH}$ is filled at the beginning of the analysis period and has to be filled up again at the end, and the total maximum volume is $98 \mathrm{GWh}$. The size of the oil storage in Case DH is calculated as a proportion of the size of the oil storage in the pulp and paper mill, where 
factors such as use of oil for electricity production are taken into account, together with the possibility to cover the oil demand for a certain number of months. The system cost for the analysed cases are calculated by adding the cost for Case DH to cases P1 - P3. P4 includes the costs for the district heating system, as both the district heating system and the pulp and paper mill are embraced by the same system boundary.

\section{Constraints - examples and comparison between the case study models}

Examples of constraints in some nodes created in the case study models are shown in Table 9. All functions stated in section 4 and described by the constraints according to Equations 5-27 can be found in the table except for Logical Equation and Function Editor, which are not included in any of the models. The investment cost function is not presented in this paper and is therefore not represented in the table, even though one such function is included. The functions implemented in reMIND can be used for different purposes and in this specific case study the Investment Cost function is included to model the variable power demand fee.

The number of different functions in the case studies, including different numbers of constraints as stated by the Equations 5-27, can be found in Table 10. The number of functions is among other things a result of the size of the analysed system, the complexity of the system, the depth of the analysis (both the total system and different parts of the system), the availability of input data for the system and the required accuracy of the results. As can be seen from the table, the dairy has more functions included in the model than the pulp and paper mill, indicating that a larger system size, such as the area of the factory site and energy use, does not imply a larger model.

Additional information regarding the size of the case study models and the time to solve the models are presented in Table 11 and, as can be seen, the solution times are modest. In the case study, ILOG CPLEX 9.0 [21] is used as optimization solver, using branch and bound as algorithm [9]. Default settings are used, except for the integrality tolerance setting for mixed integer programming (mip), which is set to 0.0 in the study (the default value is $1 * 10^{-5}$ ). The reason for this is to ensure that integers used in the model do not adopt real values, which is a possibility when using default settings. 


\section{Results and analysis of the case studies}

\subsection{Results and analysis of the dairy case study}

The system cost and the peak demand for electricity and steam are shown in Table 12. The system cost is calculated for one production year, 350 days, assuming that production over the modelled day is representative of the whole year. The total steam demand is the same for all cases while the total electricity demand is the same for all cases except Cases D5 and D6, as these have an energy efficiency measure included in the system. In addition, the electricity use in Cases D4-D6 is reduced as an absorption cooling machine is installed, operated by district heat.

Table 12 indicates increased use of the available storages in the system when including variable boundary conditions, in combination with production planning. In Case D1 the storages are not used more than necessary as there are no incentives for active operation. When facing an altered electricity price, as in Case D2, the system reacts by using the storages actively. When possible the system operates units with high electricity demand at times when the electricity price is low. Even though the average electricity price in Case D2 is equal to the constant electricity price in Case D1, there is a reduction in cost. Consequences of the altered electricity price are not only changes in production during the day and a reduction of the system cost, but also a reduction of the electrical peak demand. The electrical peak demand in Case D1 is almost $500 \mathrm{~kW}$ lower than the permitted maximum peak demand, i.e. $1500 \mathrm{~kW}$, which indicates that it might be possible to reduce the permitted maximum peak demand. This would in turn reduce the energy costs for the dairy. The electrical peak demand in Case D2 is even lower than in Case D1. This indicates that it might be possible to reduce the permitted peak even more, which may make further cost reductions possible.

When introducing a variable electrical power demand fee, as in Case D3, the system cost is even lower than in the two preceding cases. The variable electrical power demand fee, which includes a maximum power demand of $600 \mathrm{~kW}$, is almost $200 \mathrm{~kW}$ higher than the outcome of the modelling. This indicates that it might be possible to reduce the permitted maximum power demand. Consequently, the system cost may be reduced further if the contract for electrical power demand fees is negotiated. As can be seen from Table 12, the electrical power demand is reduced by more than half, when a variable electrical power demand fee is 
introduced. Compared to Case D1 the reduction is even greater. Nonetheless, the system cost is still lower than in Cases D1 and D2.

In Case D4 the possibility is introduced of using an absorption cooling machine to produce ice water. The price for district heat is fixed at a level a little higher than if ice water is produced with electricity, if the efficiencies for each cooling machine and an average electricity price are considered, and yet the absorption cooling machine is in use. The reason is the variable electrical power demand fee, adding the possibility to benefit from decreased power demand, in combination with the variable electricity price. As can be seen from Table 12, the system cost is reduced compared to the other preceding cases. Taking advantage of the flexibility in the system, i.e. using the storages, enables this. The peak for electricity demand is lower compared to the other cases, as shown in Table 12. As for the other cases, this indicates that it might be possible to reduce the permitted peak, making it possible to reduce the system cost.

The final two cases explore the possibility for the system to adapt to and benefit from the introduction of energy efficiency measures. As might be anticipated, the energy efficiency measures introduced in the system reduce the system cost, as no costs for the investment are included in the analysis. However, the size of the cost reduction is dependent on where the efficiency measure is introduced. This is due to the possibility to use the storages to different degrees. As can be seen from Table 12, the peak in electricity demand is $4 \mathrm{~kW}$ higher in Case D6 than in Case D5. Nonetheless, the system cost is lower in Case D6. The results show that the increase in the cost of electrical power in Case D6 is compensated for through production planning. If the same production plan as is Case D5 is used when introducing the efficiency measure according to Case D6, the electricity cost would decrease by 330 EUR/year. However, the electrical power demand would at the same time increase by $60 \mathrm{~kW}$, i.e. an additional cost of 1380 EUR/year. The resulting system cost in Case D6 would thus increase by 1,050 EUR/ year, compared to Case D5. Instead, using the flexibility of the system, the system cost decreases by 100 EUR/year.

For all cases the availability of storages in the model, in combination with production planning, enables the possibilities to reduce the system cost and the peak demands. The peak steam demand has not been in focus in the study. The values in Table 12 are included to check that the steam demand peaks are moderate. As a matter of fact the peaks are reduced 
compared to Case D1. It can also be seen that the steam demand peak is dependent on where an efficiency measure is introduced.

Fig. 3 shows the power demand during the day. As can be seen, it is mainly during the first time steps that the peaks are reduced.

\subsection{Results and analysis of the pulp and paper mill case study}

The system cost is shown in Table 13 and, as can be seen, introducing storage, Case P2, decreases the system cost since fuel can be purchased in months with lower prices. Electricity is produced at times with a high electricity price, which decreases the system cost even more, as can be seen in Case P3. Integration of the district heating system, Case P4, will reduce the system cost even more as the possibility to produce electricity increases with the increase in heat demand in the system.

The use of the storages is shown in Fig 4. In Cases P3, P4 and DH, the oil storage is filled up in September when oil price is lowest. When needed, most of the supplementary purchases of oil are made in February and July as the prices are relatively low in those months, and finally, in November to fill up the storage. In Case P2 oil is not used. In January and February, the bark storage is kept constantly full and is filled again in April to ensure there is enough bark in the more expensive months of May, June and July. The bark price is the same in August and September and there are therefore no differences between when to fill the storage, as reflected by the fact that Case P2 fills up in September and Cases P3 and P4 in August. In November and December the bark storage is kept constantly full.

Electricity produced in Cases P3 and P4 is shown in Fig. 5. The reason for the larger electricity production for Case P4 in the first three months and in November is the higher heat demand due to the integration of the district heating system into the analysed system. In March, electricity is produced in both cases due to the possibility to purchase fuel in February at a fairly low price. In Case P3, bark is purchased and in Case P4 both oil and bark in February. In April, electricity is produced in Case P3 but not in Case P4. The reason is that in Case P3 no extra oil needs to be purchased in February to maintain electricity production in April. In Case P4, on the other hand, extra oil needs to be purchased in February, as this is when the oil price is lowest in the early part of the year. In Case P4, no extra oil is purchased 
to maintain electricity production in April as revenues from possible electricity production in April are not enough to compensate for the cost of oil to make it profitable. Also, oil needs to be purchased in at least one more month, because the storage volume is not big enough, in order to have enough oil in the coming months when prices are higher. In Case P3, there is also some minor electricity production between May and October and in November, the reason being that there is additional capacity in the bark boiler that is used to produce steam for electricity production.

The oil demand is zero for the pulp and paper mill, when no electricity is produced at the mill or when the district heating system is not embraced by the mill system boundary. The oil demand shown for Cases P1 and P2 in Table 13 all originates from Case DH. As can be seen, when electricity production is introduced in the system oil demand increases, meaning that oil is used for electricity production (together with a small proportion of bark). When the district heating system is integrated, even more oil is used as there is a possibility to produce more electricity due to greater possibilities to deliver heat within the system.

\section{Concluding discussion}

The MIND method is a flexible method that can be adjusted according to the complexity of the analyzed system. It is the complexity of the problem and the extension of the analysis to be made that determine the resources, such as time and input data, required to construct the model and perform the analysis. An analysis can range from a simple test run to extensive studies that include, for example, multi-objective criteria, analysis of process variation, and the stability of solutions. The MIND method has been used to analyze many different aspects of industrial energy systems in several kinds of industries.

In the case studies a dairy and a pulp and paper mill are modelled and analyzed focusing important measures: fuel conversion, energy efficiency measures, load shifting, storing and system boundary expansion. These measures may be related to the issues stated by the European Commission [1,2], briefly described in the introduction, in different ways. Fuel conversion, as stated in the dairy case study, reduces the use of electricity and increases the use of district heat. Depending on the origin of the electricity and district heat, the issues regarding security of supply, the reduction of $\mathrm{CO}_{2}$ emissions (global warming issue), and an increased level of renewables are targeted. 
550 Generally speaking, an energy efficiency measure targets reduction of global primary energy use. In the dairy case study, the electricity demand is reduced by introducing an energy efficient process and, depending on the fuel used to produce the marginal electricity in the system both the potential to reduce $\mathrm{CO}_{2}$ emissions (global warming issue) and greater security of supply can be estimated.

Load shifting and storing support security of supply, for example storing energy as in the pulp and paper mill case study, have the possibility to level out the risk of possible interruptions in delivery. Load shifting, as in the dairy case study, helps to moderate the risk of power demand deficit. Load shifting and storing may also have an impact on the possibility to reduce $\mathrm{CO}_{2}$ emissions (global warming issue) and the use of renewables, since in the dairy case study, for example, it is likely that a reduction in peak loads will affect the mix of fuels used to produce the electricity. This is a result of all types of electricity production units often being needed to keep the system in balance during peak periods and the ones emitting most $\mathrm{CO}_{2}$ are thus also in use. When producing electricity during low-demand hours, production units emitting less $\mathrm{CO}_{2}$ can be used.

In the pulp and paper mill case study the fifth measure, a system boundary expansion, is introduced. As shown in the case study, more oil is used because more electricity is produced within the system. Hence, the possibility to reduce the impact of global warming, due to expansion of the system boundary, is dependent on how $\mathrm{CO}_{2}$ emissions from electricity production in the total system are calculated. If it is assumed that coal condensing power plants produce the electricity that is replaced by the electricity in the case study, a positive effect on emissions of $\mathrm{CO}_{2}$ (global warming issue) is found, as the $3 \mathrm{GWh}$ of extra oil are needed to produce $3 \mathrm{GWh}$ of electricity, implying a much better efficiency than the worst coal condensing plants in the system. The same line of thought may be used when estimating the possibility to reduce the use of primary energy. Moreover, when expanding the system boundary it is possible to invest in larger steam producing units, as the heat demand increases, and the efficiency of boilers combusted with renewables are improved with increased size and consequently it is more profitable to invest in such boilers, which in the end increase the level of renewable energy. A system boundary expansion can also improve the security of supply if the renewables are produced close to the studied system. 
In addition to this, the primary goal of the optimizations is to minimize energy costs and thus improve the overall competitiveness of the dairy and the pulp and paper mill, which is the first issue in [1]. The results show how the different measures reduce, for example, the system costs, peaks in the electricity and steam demand, and how storages are used.

The case study model of the dairy is first used to picture how future characteristics of electricity prices may influence the system. Traditionally, electricity prices in Sweden have varied mainly over the seasons, but Sweden is now facing more continental electricity prices, i.e. with fluctuations over the day [33]. Using the MIND method, the case study shows that it is possible to profit from such a differentiated price.

Peaks in the electricity demand may generate congestion problems in the grid, but also a power deficit in extreme cases [34]. Following the deregulation of the electricity market in the European Union [35], the margin between ordinary generation capacity and electricity demand has narrowed and the amount of standby power production has been limited in Sweden; load shifting is one alternative to reduce the problem [34]. Power demand fees may be used for load shifting and one alternative is tested in this paper, using the MIND method. The dairy case study shows that a variable power demand fee may be used to reduce the power demand in a company like the one studied, and also benefit from such an arrangement.

In Sweden the reductions in the use of electricity are promoted in, for example, a programme launched by the Swedish energy agency [36]. Replacing a compressor-driven cooling machine with an absorption cooling machine is one way. Using the MIND method, such changes can be analysed and the results from the case study indicate possible gains. Another way is to implement energy efficiency measures. As shown in the dairy case study, it is possible to use the MIND method to analyse these kinds of changes. As can be seen from the case study, the MIND method may be used to discover outcomes that are not easy to predict, such as that both the size of the peak in electricity demand and the system cost are dependent on where the efficiency measure is introduced.

The MIND method was originally and primarily developed as a tool for researchers, but is also used for educational purposes. The models used in the case studies are constructed as a part of a previous research project, and subsequently validated, and have later been modified and simplified to be suitable for educational purposes to show students how different 
measures affect an industrial energy system. Therefore, there are some simplifications in the models. For example, costs for start-ups and stops are not included. A model, such as the dairy model, reproducing 24 hours may also not be representative as some products might be prepared the day before and/or delivered the day after. Also, power demand fees are difficult to reproduce exactly when only looking at one day. An extension of the dairy case study is to analyze a whole year to reproduce the dairy more correctly, but this requires more input data to be provided by the staff at the dairy, whose time available for collecting and compiling the data is often limited due to slimmed organizations. Also, as shown, the solution times for the models included in this study are moderate. Expanding the models, both regarding the length of the analysis period and the level of detail, implies that computer solution times will increase. The purpose of the study must always determine the size of the model and the main aim of this paper, i.e. to show the effect of the introduction of different measures in industrial energy systems in relation to the issues stated by the European Commission [1,2], is accomplished using the models presented in the case studies by using the MIND method for such analyses.

\section{Acknowledgements}

Peter Blomqvist (former Sandberg) is acknowledged for his important contributions of the early drafts of this paper and Bahram Moshfegh is acknowledged for ideas regarding the focus of the paper. Thanks are also due to everyone involved in the development of the reMIND software, including those who performed the actual Java coding and all users of the program for their queries and their opinions, especially Nawzad Mardan at the Energy System division at Linköping University and Mikael Larsson at MEFOS.

\section{References}

[1] COM (Commission of the European Communities). Green Paper on energy efficiency of doing more with less, ISBN 92-894-9819-6. European Communities, 2005.

[2] COM (Commission of the European Communities). 30 final. 2020 by 2020, Europe's climate change opportunity. Brussels, 2008. 
[3] Simon HA. Administrative behavior - A study of decision-making processes in administrative organizations. The Free Press: New York, USA; 1997.

[4] Sandberg P, Söderström M. Industrial energy efficiency - the need for investment decision support from a manager perspective. Energy Policy 2003;31: 1623-1634.

[5] Ingelstam L. Systems - considering society and technology (System - att tänka över samhälle och teknik (In Swedish)). EB:1:2002: Eskilstuna, Sweden; 2002.

[6] Amelin M. On Monte Carlo Simulation and Analysis of Electricity Markets. Dissertation, Royal Institute of Technology: Stockholm, Sweden; 2004.

[7] Taylor JG. Mathematical Approaches to Neural Networks. North-Holland: Amsterdam; 1993.

[8] Box GEP, Hunter JS, Hunter WG. Statistics for experimenters. Wiley: New York; 1978.

[9] Rardin RL. Optimization in operations research. Prentice-Hall Inc.: New Jersey, USA; 1998.

[10] Taha HA. Operation research - An introduction. Macmillan Publishing Co., Inc.: New York, USA; 1976.

[11] Grossmann IE, Santibanez J. Applications of mixed-integer linear programming in process synthesis, Computers \& Chemical Engineering 1980;4 (4).

[12] Grossmann IE, Caballero JA, Yeomans H. Advances in Mathematical Programming for Automated Design, Integration and Operation of Chemical Processes. Proceedings of the International Conference on Process Integration: Copenhagen, Denmark; 1999.

[13] Kong H, Qi E, Li H, Li G, Zhang X. An MILP model for optimization of byproduct gases in the integrated iron and steel plant, Applied Energy, doi:10.1016/j.apenergy.2009.11.031, 2010. 
[14] Chae SH, Kim SH, Yoon S-G, Park S. Optimization of a waste heat utilization network in an eco-industrial park, Applied Energy, doi:10.1016/j.apenergy.2009.12.003, 2010

[15] Karlsson M, Söderström M. Sensitivity analysis of investments in the pulp and paper industry. International Journal of Energy Research 2002;26: 1253-1267.

[16] Larsson M, Sandberg P, Dahl J, Söderström M, Vourinen H. System profits of widening the system boundaries - renovation of the coke oven battery at an integrated steel plant. International Journal of Energy Research 2004;28: 1051-1064.

[17] Wolf A, Karlsson M. Evaluating the environmental benefits of industrial symbiosis: discussion and demonstration of a new approach, Progress in Industrial Ecology - An International Journal, 2008;5: 502-517.

[18] Nilsson K. Cost-Effective Industrial Energy Systems - Multiperiod Optimization of Operating Strategies and Structural Choices. Linköping Studies in Science and Technology, Dissertation No. 315, Linköping University: Linköping, Sweden; 1993.

[19] Metcalf M, Reid J. FORTRAN 90/95 explained. Oxford University Press Inc.: New York, USA; 1999.

[20] Flanagan D. Java in a nutshell. O’Reilly \& Associates, Inc., Sebastopol: CA, USA; 1999.

[21] CPLEX. Using the CPLEX Callable Library. CPLEX Optimization Inc, Incline Village: NV, USA; 1995.

[22] lp_solve, Open source (Mixed Integer) Linear Programming system. Co-developers: Michel Berkelaar, Kjell Eikland and Peter Notebaert, Release data: Version 5.1.0.0 dated 1 May 2004, http://lpsolve.sourceforge.net/, last accessed March 31, 2010.

[23] Thollander P, Mardan N, Karlsson K. Optimisation as investment decision support in a Swedish medium-sized iron foundry - a move beyond traditional energy auditing, Applied Energy, 2009;86: 433-440. 
[24] Sandberg P, Larsson M, Dahl J, Söderström M, Vourinen H. In search of Stability investigating flexible and stable production strategies for an optimised steel plant. Scanmet II.

Proceedings of the $2^{\text {nd }}$ international Conference on Process Development in Iron and Steelmaking: Sweden; 2004.

[25] Karlsson M. A systems approach to the reduction of oil demand in a Swedish board mill. Energy - The International Journal 2004;29: 103-124.

[26] Bengtsson C, Karlsson M, Berntsson T, Söderström M. Co-ordination of Pinch Technology and the MIND method - applied to a Swedish board mill. Applied Thermal Engineering 2002;22: 133-144.

[27] Larsson M, Sandberg P. Analysing the Influence of Variations when Optimising the Energy and Material System for an Integrated Steel Plant. Proceedings of the $4^{\text {th }}$ International Conference on Fluid and Thermal Energy Conversion, FTEC 2003: Indonesia; 2003.

[28] Gong M, Karlsson M. Co-ordination of Exergy Analysis and the MIND Method Applied to a Pulp and Board Mill, International Journal of Exergy 2004;1: 289-302.

[29] Andersson J. A survey of multi-objective optimisation in engineering design. Technical report LiTH-IKP-R-1097: Linköping, Sweden; 2000.

[30] Heidari Tari M, Söderström M. Modeling of thermal energy storage in industrial energy systems the method development of MIND. Applied Thermal Engineering 2002;22: 11951205.

[31] Heidari Tari M, Söderström M. Optimisation modelling of industrial energy systems using MIND introducing the effect of material storage. International Journal of Operational Research 2002;142: 419-433.

[32] Karlsson M., Mardan N. Timing and sizing of investments in industrial processes - the use of an optimization tool. Submitted to ECOS conference, 2010 (Unpublished results) 
749 [33] Melkersson M, Söderberg S-O. (Dynamic electricity prices - pricing in a integrated

750 European electricity market (Dynamiska elpriser - elprissättning på en integrerad europeisk

751 elmarknad, (in Swedish)). LiTH-IKP-Ex-2114, Linköping University: Linköping, Sweden;

7522004.

[34] SvK (Svenska Kraftnät) (Swedish Transmission System Operator). Power supply at peaks (Effekttillgång vid höglast (in Swedish)). Vällingby, Sweden; 2004.

[35] COM (Commission of the European Communities). 125 final, Proposal for a directive of the European Parliament and of the Council amending Directives 96/92/EC and 98/30/EC concerning common rules for the internal market in electricity and natural gas. Brussels;

\section{1.}

[36] Swedish statutes. Law on programmes for energy efficiency measures (Lag om program för energieffektiviseringsåtgärder (in Swedish)). SFS2004:1196: Sweden; 2004. 


\section{Nomenclature}

Parameters

a coefficient representing a normalizing factor for the objective function (real) a coefficient representing a weighting factor for the objective function (real) a coefficient representing e.g.: (1) a slope of a function [slope], (2) a step in a function [step] and (3) a minimum [min] or maximum [max] value of a variable (real)

flow entering a node (integer)

total number of flows entering a node (integer)

flow leaving a node (integer)

total number of flows leaving a node (integer)

a flow (integer)

total number of objective types (integer)

represents the total flow for a specific flow for all time steps included. a slope within the function (integer) $(\mathrm{p}=0$ represents the point when the larger than zero) total number of slopes within a function (integer) the number of time steps the adjusting time needs to be in operation for a batch process (integer) total number of adjusting time steps for a batch process (integer) time step (integer) total amount of time steps (integer) the number of a specific real variable the total number of real variables in the problem the number of a specific integer variable the total number of integer variables 
$799 \quad z$

$800 \quad Z$

$801 \quad B T$

$802 \quad M S T$

$803 \eta$

804

805

806

Variables

$807 x$

$808 \quad Y$

$809 \quad F R$

810 IC

$811 S E$

the number of a specific constraint (integer)

total number of constraints (integer)

the number of time steps a batch needs to stay put (integer)

maximum allowable time steps to store a subject (integer)

efficiency: (1) entering storage (2) leaving the storage or (3) efficiency of the storage (real)

$812 \lambda$

813

real variable, represents a flow of any kind

binary variable (only attaining the values 0 or 1 )

real variable in the function FlowRelation

real variable representing the investment cost in the function InvestmentCost

real variable representing the storage volume in the function StorageEquation 


\section{Captions}

\section{Figures}

Fig. 1. User interface of the decision support system reMIND, showing the dairy case study model (explained in 5.1).

Fig. 2. The case study model of the pulp and paper mill.

Fig. 3. Electrical power demand for each hour during the modelled day in the case study of the dairy.

Fig. 4. Stored volume for the bark and oil storages in the case study of the pulp and paper

Fig. 5. Electricity produced in the case study of the pulp and paper mill .

Tables

Table 1. Input data for the processes in the dairy in the model.

Table 2. Summary of the different analyzed cases at the dairy.

Table 7. Prices and district heating demand used in the model ${ }^{1}$.

Table 3. Summary of prices and fees for the different dairy cases.

Table 4. Variable price of electricity in the dairy case study [EUR/MWh]. Average: 42.2 EUR/MWh.

Table 5. Input data for the processes in the pulp and paper mill in the model.

Table 6. Input data for the boilers in the pulp and paper mill in the model ${ }^{1}$. 
Table 8. Summary of the different analyzed cases at the pulp and paper mill.

Table 9: Examples of constraints in 6 nodes in the cases studied, showing the constraints for Time step t. P indicates that the constraints are taken from the pulp and paper mill model and D indicates that the constraints are taken from the dairy model. ${ }^{1}$ Each flow, found in the table, are found in Figs. 1 and 2.

Table 10: Number of different functions in the cases studied. The intervals stated in the table are due to the different cases studied at the dairy and the pulp and paper mill, respectively.

Table 11: Information about the size and solution times for the case studies. The intervals stated in the table are due to the different cases studied at the dairy and the pulp and paper 860 mill, respectively.

Table 12. System cost and peak demand at the dairy.

Table 13. System cost, oil and bark demand and electricity production at the pulp and paper 865 mill 


\section{gure 1}

Click here to download high resolution image

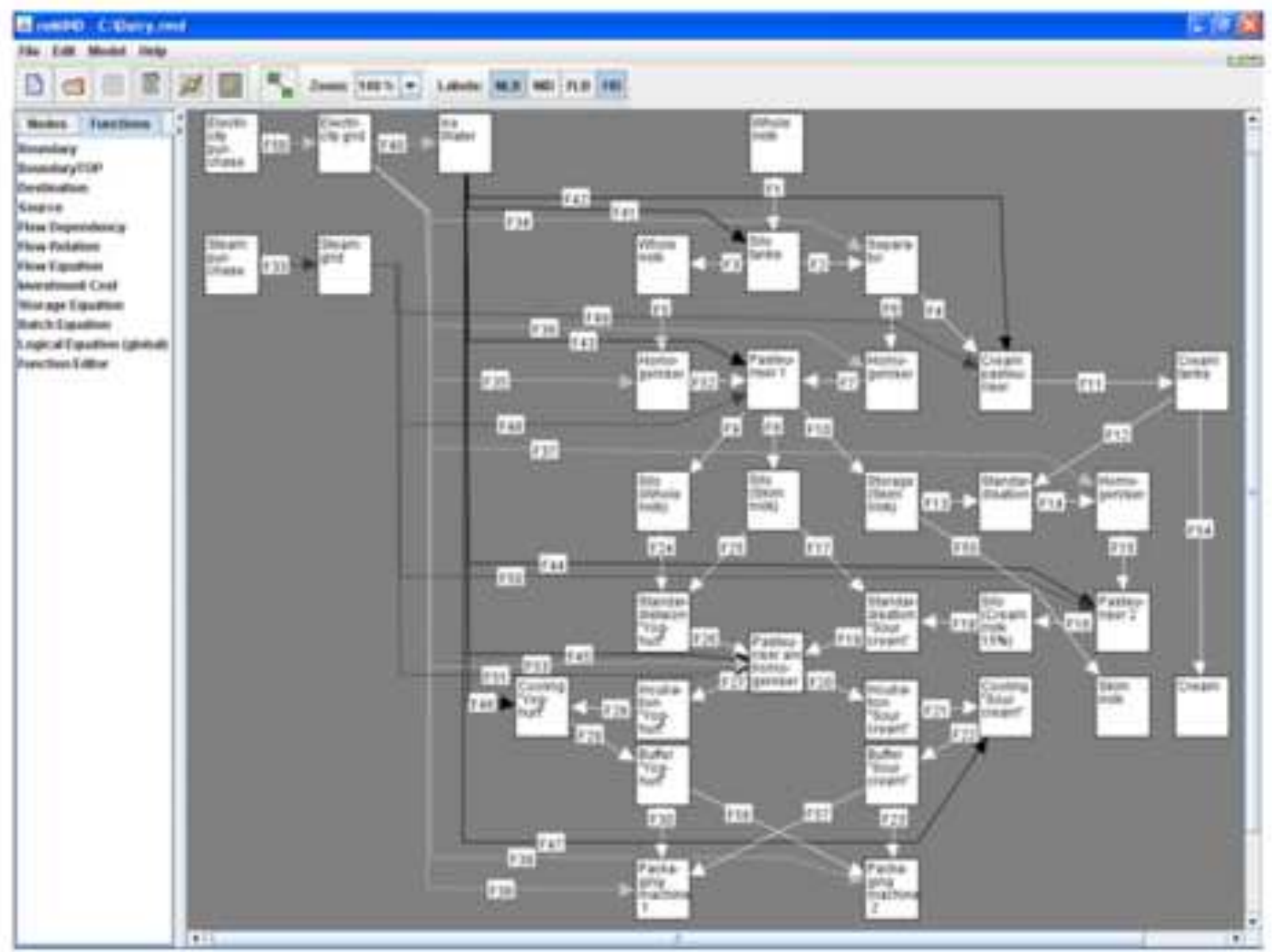




\section{gure 2}

Click here to download high resolution image

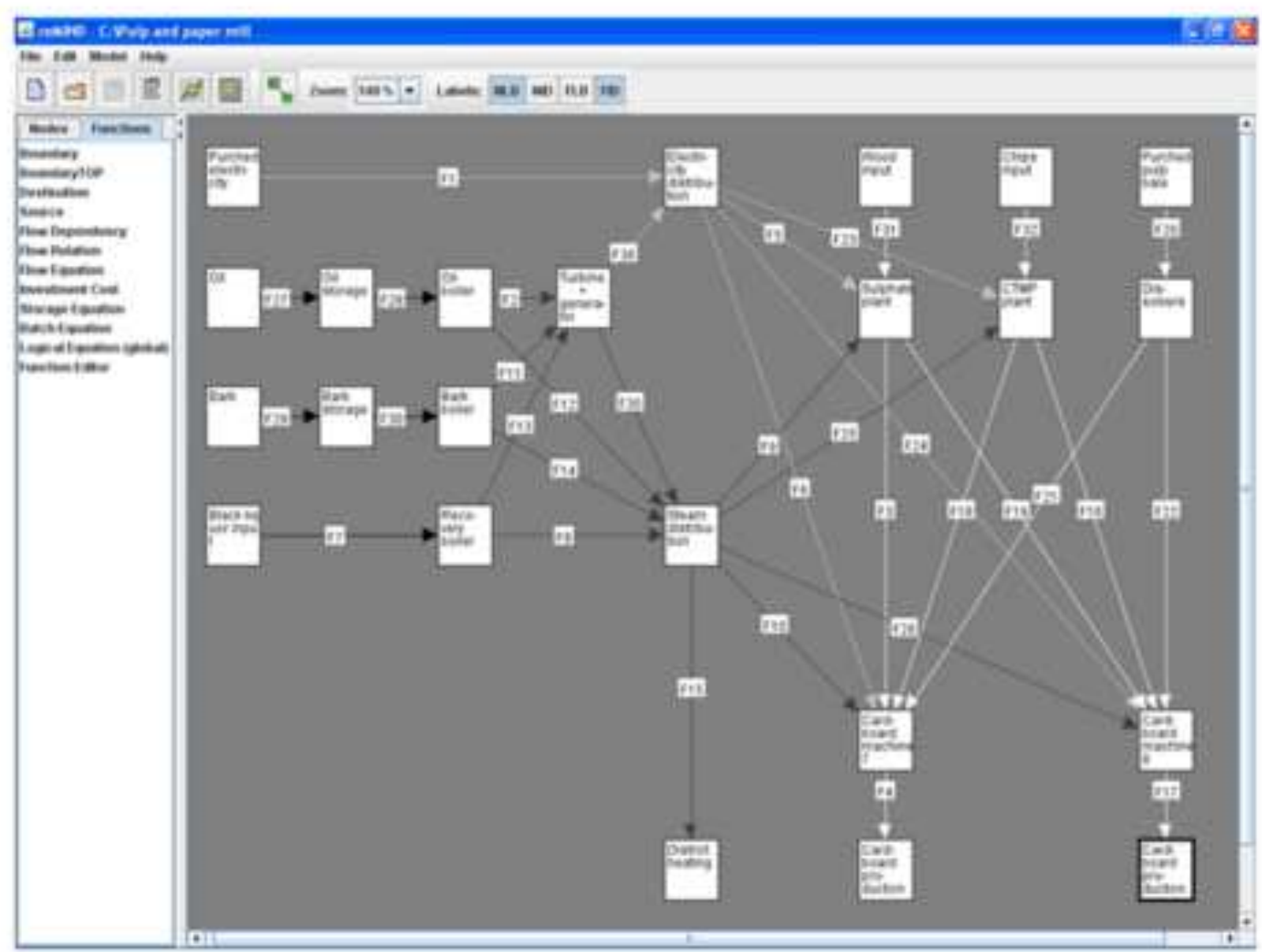




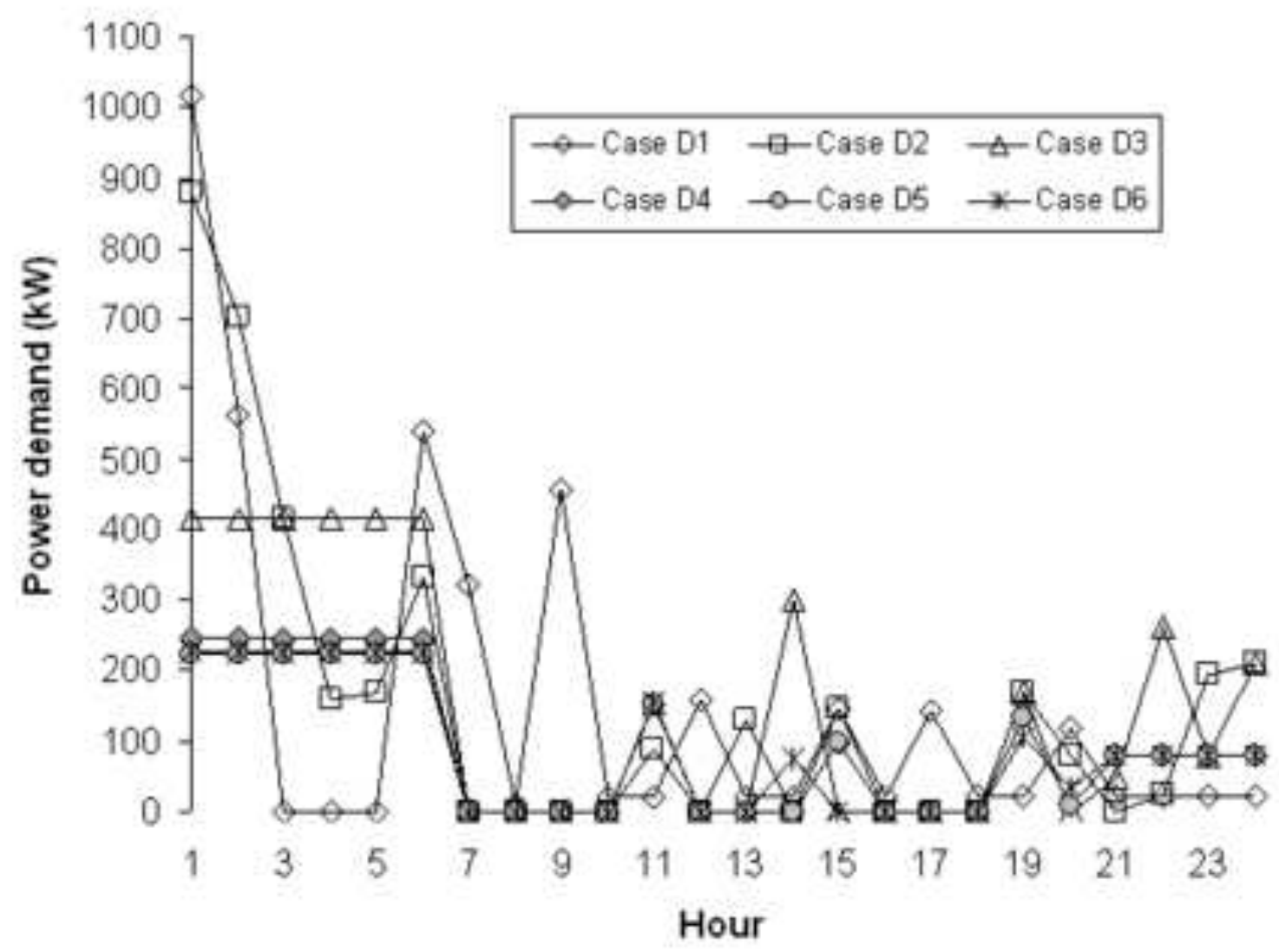




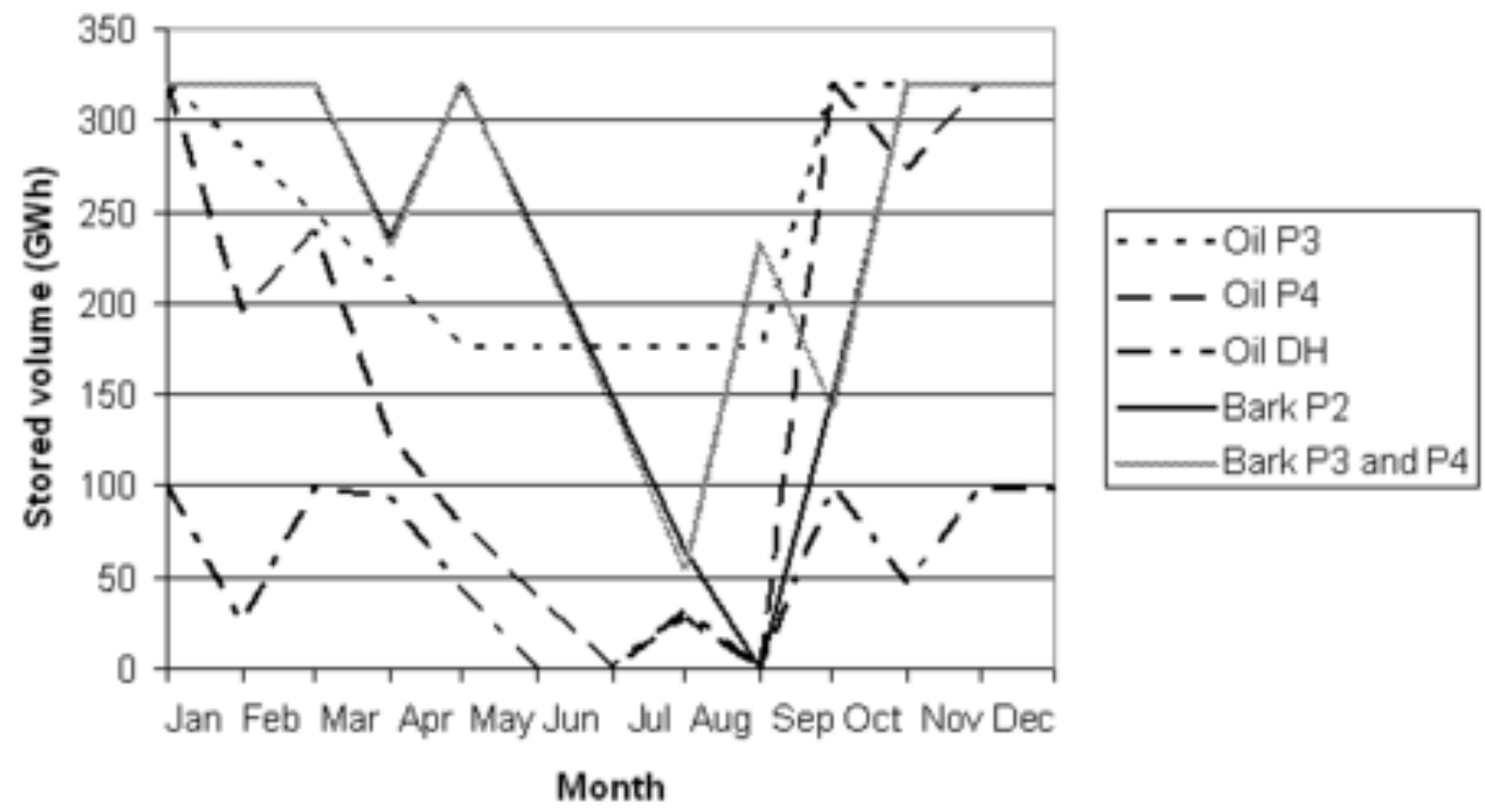




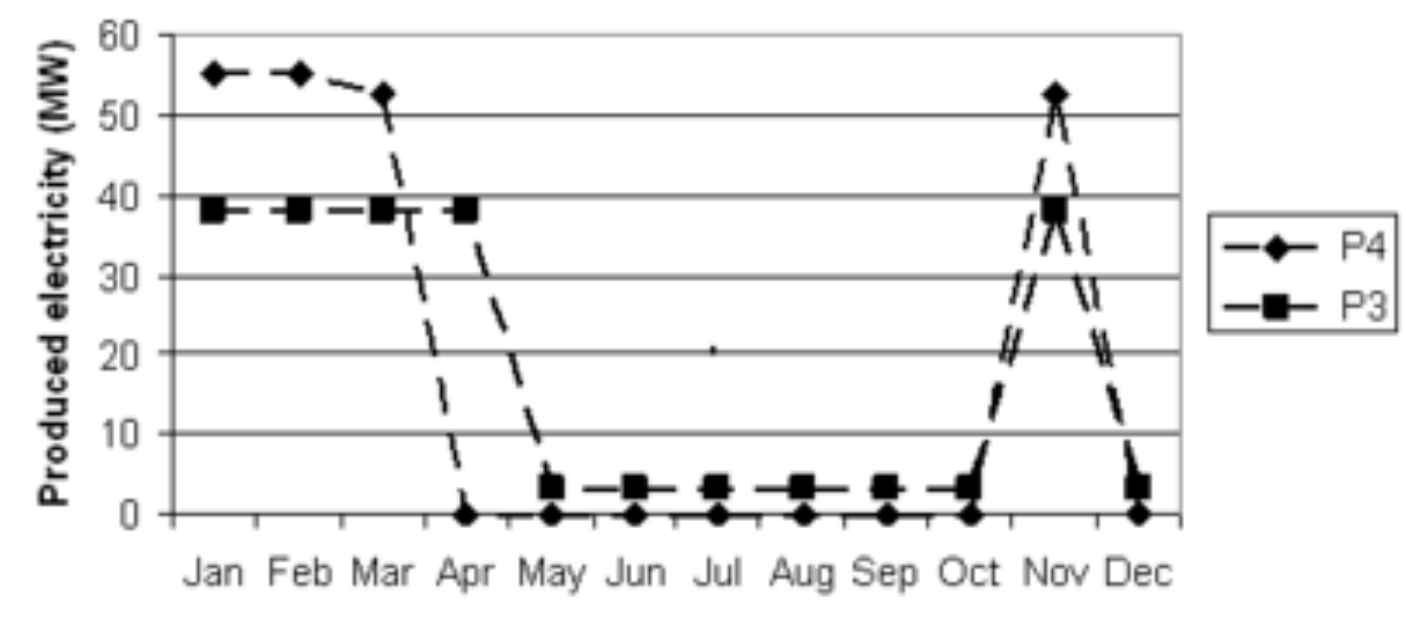

Month 
Table 1. Input data for the processes in the dairy model.

\begin{tabular}{|c|c|c|c|c|c|}
\hline & $\begin{array}{l}\text { Electricity } \\
\left(\mathrm{kWh} / \mathrm{m}^{3}\right)\end{array}$ & $\begin{array}{c}\text { Steam } \\
\left(\mathrm{kWh} / \mathrm{m}^{3}\right)\end{array}$ & $\begin{array}{l}\text { Ice water } \\
\left(\mathrm{kWh} / \mathrm{m}^{3}\right)\end{array}$ & $\begin{array}{c}\text { Max } \\
\text { production } \\
\left(\mathrm{m}^{3}\right)\end{array}$ & $\begin{array}{l}\text { Incubation time/ } \\
\text { max storage time } \\
\text { (h) }\end{array}$ \\
\hline Silo tanks & - & - & 4.66 & - & 20 \\
\hline Separator ${ }^{\mathrm{a}}$ & 1.049 & - & - & - & - \\
\hline Homogenizer & 9.2 & - & - & - & - \\
\hline Pasteurizer 1 & - & 5.2 & 7.0 & 40 & - \\
\hline Cream pasteurizer & - & 14.0 & 70.0 & 7.5 & - \\
\hline Cream tanks & - & - & - & - & 15 \\
\hline Pasteurizer 2 & - & 38.0 & 14.0 & 20.0 & - \\
\hline Silo (Cream milk) & - & - & - & - & 15 \\
\hline Silo (Whole milk) & - & - & - & - & 15 \\
\hline Silo (Skim milk) & - & - & - & - & 15 \\
\hline Incubation (Yoghurt) ${ }^{\mathrm{b}}$ & - & - & - & - & 5 \\
\hline Incubation (Sour cream) ${ }^{\mathrm{b}}$ & - & - & - & - & 8 \\
\hline Pasteurizer and homogenizer & 9.2 & 38.0 & 7.0 & 20.0 & - \\
\hline Cooling (Yoghurt) & - & - & 25.7 & - & - \\
\hline Cooling (Sour cream) & - & - & 25.7 & - & - \\
\hline Buffer (Yoghurt) & - & - & - & - & 6 \\
\hline Buffer (Sour cream) & & & & & 15 \\
\hline Packaging machine 1 & 5.5 & - & - & 6.06 & - \\
\hline Packaging machine 2 & 5.5 & - & - & 8.4 & - \\
\hline
\end{tabular}

${ }^{\mathrm{a}} 10.39 \%$ of the whole milk is separated as cream and the rest is skim milk.

${ }^{\mathrm{b}}$ In the model there is no production of yoghurt in the packaging machines during the first 5 hours and no production of sour cream in the packaging machines during the first 8 hours. This is due to the fact that the incubation times for these products are 5 and 8 hours, respectively. 
Table 2. Summary of the different analyzed cases at the dairy.

\begin{tabular}{lccccc}
\hline Case & $\begin{array}{c}\text { Price of } \\
\text { electricity }\end{array}$ & $\begin{array}{c}\text { Electrical power } \\
\text { demand fee }\end{array}$ & $\begin{array}{c}\text { Absorption } \\
\text { cooling } \\
\text { machine }^{\mathrm{a}}\end{array}$ & $\begin{array}{c}\text { Energy efficiency } \\
\text { measure }\end{array}$ & $\begin{array}{c}\text { Production } \\
\text { planning }\end{array}$ \\
\hline D1 & Constant & Constant & None & None & Constant \\
D2 & Variable & Constant & None & None & Variable \\
D3 & Variable & Variable & None & None & Variable \\
D4 & Variable & Variable & District heat & None & Variable \\
D5 & Variable & Variable & District heat & $\begin{array}{c}\text { Implemented } \\
\text { Implemented }\end{array}$ & Variable \\
D6 & Variable & Variable & District heat & Variable \\
\hline
\end{tabular}

${ }^{\mathrm{a}}$ The electrically powered compressor to produce ice water, with an efficiency of 2.8 , has been exchanged for an absorption cooling machine powered by district heat, with an efficiency of 0.8

${ }^{b}$ The electricity demand for one of the homogenizers is reduced to half the original demand. This homogenizer is situated at the beginning of the production line in Case D5 and at the end of the production line in Case D6. 
Table 3. Summary of prices and fees for the different dairy cases.

\begin{tabular}{lll}
\hline & Constant [EUR/MWh] & Variable \\
\hline Price of electricity & $42.2 \mathrm{EUR} / \mathrm{MWh}$ & See Table 4 (Average 42.2 EUR/MWh) \\
Electrical power demand fee & $23,330 \mathrm{EUR} / \mathrm{year}^{\mathrm{a}, \mathrm{c}}$ & $23.3 \mathrm{EUR} / \mathrm{kW}^{\mathrm{b}, \mathrm{c}}$ \\
Price of steam & $43.1 \mathrm{EUR} / \mathrm{MWh}^{\mathrm{d}}$ & - \\
Price of district heating & $12.3 \mathrm{EUR} / \mathrm{MWh}^{\mathrm{d}}$ & - \\
\hline
\end{tabular}

\footnotetext{
${ }^{\mathrm{a}}$ The maximum peak demand 1,500 MW.

${ }^{\mathrm{b}}$ A constant amount of 9,350 EUR/year is also included. The variable electrical power demand fee is estimated to amount to the fixed fee, i.e. 23,330 EUR/year, when a maximum peak demand of $600 \mathrm{~kW}$ is included.

${ }^{\mathrm{c}}$ If the maximum peak demand is exceeded there is penalty fee of $100 \mathrm{EUR} / \mathrm{kW}$.

${ }^{\mathrm{d}}$ No power limit.
} 
Table 4. Variable price of electricity in the dairy case study [EUR/MWh]. Average: 42.2 EUR/MWh.

\begin{tabular}{lcccccccccccc}
\hline Hour & $\mathbf{1}$ & $\mathbf{2}$ & $\mathbf{3}$ & $\mathbf{4}$ & $\mathbf{5}$ & $\mathbf{6}$ & $\mathbf{7}$ & $\mathbf{8}$ & $\mathbf{9}$ & $\mathbf{1 0}$ & $\mathbf{1 1}$ & $\mathbf{1 2}$ \\
Electricity price & 32.2 & 32.2 & 32.2 & 32.2 & 32.2 & 38.9 & 51.1 & 51.1 & 47.8 & 46.7 & 47.8 & 48.9 \\
Hour & $\mathbf{1 3}$ & $\mathbf{1 4}$ & $\mathbf{1 5}$ & $\mathbf{1 6}$ & $\mathbf{1 7}$ & $\mathbf{1 8}$ & $\mathbf{1 9}$ & $\mathbf{2 0}$ & $\mathbf{2 1}$ & $\mathbf{2 2}$ & $\mathbf{2 3}$ & $\mathbf{2 4}$ \\
Electricity price & 50.0 & 48.9 & 48.9 & 51.1 & 51.1 & 50.0 & 44.4 & 38.9 & 37.8 & 34.4 & 32.2 & 32.2 \\
\hline
\end{tabular}


Table 5. Input data for the processes in the pulp and paper mill model.

\begin{tabular}{|c|c|c|c|c|c|c|}
\hline & $\begin{array}{l}\text { Electricity } \\
\left(\mathrm{MWh} / \mathrm{t}_{90}{ }^{\mathrm{a}}\right)\end{array}$ & $\begin{array}{c}\text { Steam }^{\mathrm{b}} \\
\left(\mathrm{MWh} / \mathrm{t}_{90}{ }^{\mathrm{a}}\right)\end{array}$ & Efficiency & $\begin{array}{c}\text { Amount of } \\
\text { Sulphate pulp } \\
(\%)\end{array}$ & $\begin{array}{l}\text { Amount of } \\
\text { CTMP } \\
(\%)\end{array}$ & $\begin{array}{c}\text { Amount of } \\
\text { Pulp bale } \\
(\%)\end{array}$ \\
\hline Sulphate plant $^{\mathrm{d}}$ & 0.71 & 1.96 & $4.65^{\mathrm{e}}$ & - & - & - \\
\hline CTMP plant ${ }^{\mathrm{f}}$ & 1.46 & 0.18 & $2.25^{\mathrm{g}}$ & - & - & - \\
\hline Cardboard machine 7 & 0.66 & 1.69 & $85^{\mathrm{h}}$ & $40-60$ & $25-50$ & $>2$ \\
\hline Cardboard machine 8 & 0.96 & 1.82 & $85^{\mathrm{h}}$ & $44-53$ & $30-45$ & $>2$ \\
\hline
\end{tabular}

${ }^{\mathrm{a}} \mathrm{t}_{90}$ is the weight for pulp or cardboard with a dryness of $90 \%$.

${ }^{\mathrm{b}}$ The mill case study has two different steam pressure levels for deliveries to the processes: 4 bar and 11 bar. For simplification reasons, only one pressure level, 4 bar, is considered in this case study. The input data is the sum of the two different steam pressure levels.

${ }^{\mathrm{c}}$ The part of the pulp demand that is purchased is delivered as bales from a pulp mill and is dissolved in a pulp dissolver. The energy demand for the dissolver is ignored in this case study as it is a minor demand compared to the demand in the other processes.

${ }^{\mathrm{d}}$ In the sulphate plant the screening, the digester, washing, the lime kiln and bleaching are included.

${ }^{\mathrm{e}}$ This efficiency defines how many $\mathrm{m}^{3}$ sub ( $\mathrm{sub}=$ solid under bark, i.e. the amount of wood that is located beneath the bark of a tree trunk) are needed to produce one tonne pulp with a dryness of $90 \%\left(\mathrm{t}_{90}\right)$.

${ }^{\mathrm{f}}$ In the CTMP plant some steam is produced that is delivered to the steam grid. This is excluded in this case study.

${ }^{\mathrm{g}}$ This efficiency defines how many piled $\mathrm{m}^{3}$ of chips are needed to produce one tonne pulp with a dryness of $90 \%\left(t_{90}\right)$.

${ }^{\mathrm{h}}$ The percentage of the pulp delivered to the cardboard machines that produce cardboard. The rest is discharged as reject and trim losses. These are taken care of by being ground and then included in some cardboard qualities. In this case study, this "recycling system" is considered by reducing the percentage share of pulp bale. 
Table 6. Input data for the boilers in the pulp and paper mill model ${ }^{\mathrm{a}}$.

\begin{tabular}{lcc}
\hline & $\begin{array}{c}\text { Maximum output } \\
(\mathrm{MW})\end{array}$ & $\begin{array}{c}\text { Efficiency } \\
(\%)\end{array}$ \\
\hline Oil boiler & 210 & 92 \\
Bark boiler & 110 & $87^{\mathrm{b}}$ \\
Recovery boiler & $110^{\mathrm{c}}$ & 66 \\
\hline
\end{tabular}

${ }^{\text {a }}$ A destruction oven, where gases are combusted, that delivers steam to the steam grid is not considered in this case study as it is a minor delivery compared to the other boilers.

${ }^{\mathrm{b}}$ The efficiency for the bark boiler varies due to the moisture in the fuel, but is approximated in this case study.

${ }^{\mathrm{c}}$ The production of steam in the recovery boiler depends on the production of sulphate pulp. At the production rate that is predetermined in this case study the recovery boiler delivers $110 \mathrm{MW}$. Except black liquor, some minor proportion of electricity and oil are used in the recovery boiler, which is not considered in this case study. 
Table 7. Prices and district heating demand used in the model ${ }^{\mathrm{a}}$.

\begin{tabular}{lllllllllllll}
\hline & Jan & Feb & Mar & Apr & May & Jun & Jul & Aug & Sep & Oct & Nov & Dec \\
\hline Electricity price (EUR/MWh) & 54 & 52 & 48 & 43 & 38 & 28 & 28 & 36 & 39 & 42 & 47 & 52 \\
Oil price (EUR/MWh) & 33 & 31 & 36 & 37 & 39 & 34 & 30 & 31 & 28 & 32 & 31 & 38 \\
Bark price (EUR/MWh) & 9 & 10 & 11 & 10 & 11 & 12 & 11 & 10 & 10 & 9 & 11 & 12 \\
District heating demand (MW) & 96 & 96 & 82 & 68 & 55 & 55 & 27 & 41 & 55 & 68 & 82 & 96
\end{tabular}

${ }^{\mathrm{a}}$ The price for the black liquor is set to 0 in the model as it is a part of the chemical recovery cycle. 
Table 8. Summary of the different analyzed cases at the pulp and paper mill.

\begin{tabular}{lccc}
\hline Case & Storage & $\begin{array}{c}\text { Electricity } \\
\text { production at site }\end{array}$ & $\begin{array}{c}\text { Including } \\
\text { district heating } \\
\text { system }\end{array}$ \\
\hline P1 & None & None & No \\
P2 & Included & None & No \\
P3 & Included & Included & No \\
P4 & Included & Included & Yes \\
DH & - & - & - \\
\hline
\end{tabular}


Table 9: Examples of constraints in 6 nodes in the cases studied, showing the constraints for Time step $t$. P indicates that the constraints are taken from the pulp and paper mill model and $\mathrm{D}$ indicates that the constraints are taken from the dairy model. Each flow in the table can be found in Figs. 1 and 2.

\begin{tabular}{|c|c|c|}
\hline Node & Function & Constraints \\
\hline Purchased electricity $(\mathrm{P})$ & Source & $\begin{array}{l}54 * \mathrm{~F} 1_{1} ; 52 * \mathrm{~F} 1_{2} ; 48 * \mathrm{~F} 1_{3} ; 43 * \mathrm{~F} 1_{4} ; 38 * \mathrm{~F} 1_{5} ; 28 * \mathrm{~F} 1_{6} ; \\
28 * \mathrm{~F} 1_{7} ; 36 * F 1_{8} ; \quad 39 * \mathrm{~F} 1_{9} ; \quad 42 * \mathrm{~F}_{10} ; \quad 47 * \mathrm{~F} 1_{11} ; \\
52 * \mathrm{~F} 1_{12}\end{array}$ \\
\hline Oil boiler $(\mathrm{P})$ & Flow Dependency & $0.92 * \mathrm{~F} 28_{\mathrm{t}}=\mathrm{F} 2_{\mathrm{t}}+\mathrm{F} 12_{\mathrm{t}}$ \\
\hline Oil boiler $(\mathrm{P})$ & Boundary & $\mathrm{F} 22_{\mathrm{t}}+\mathrm{F} 12_{\mathrm{t}}<=210$ \\
\hline Cardboard machine $8(\mathrm{P})$ & Flow Relation & $\begin{array}{l}\mathrm{F} 16_{\mathrm{t}}+\mathrm{F} 19_{\mathrm{t}}+\mathrm{F} 22_{\mathrm{t}}=\mathrm{FR}_{\mathrm{t}} \\
\mathrm{F} 16_{\mathrm{t}}<=0.45 * \mathrm{FR}_{\mathrm{t}} \\
\mathrm{F} 16_{\mathrm{t}}>=0.35 * \mathrm{FR}_{\mathrm{t}} \\
\mathrm{F} 19_{\mathrm{t}}<=0.53 * \mathrm{FR}_{\mathrm{t}} \\
\mathrm{F} 19_{\mathrm{t}}>=0.43 * \mathrm{FR}_{\mathrm{t}} \\
\mathrm{F} 22_{\mathrm{t}}>=0.02 * \mathrm{FR}_{\mathrm{t}}\end{array}$ \\
\hline Cardboard machine $8(\mathrm{P})$ & Flow Dependency & $\mathrm{F} 16_{\mathrm{t}}+\mathrm{F} 19_{\mathrm{t}}+\mathrm{F} 22_{\mathrm{t}}=0.85^{*} \mathrm{~F} 17_{\mathrm{t}}$ \\
\hline Cardboard machine $8(\mathrm{P})$ & Flow Dependency & $\mathrm{F} 24_{\mathrm{t}}=0.96 * \mathrm{~F} 17_{\mathrm{t}}$ \\
\hline Cardboard machine $8(\mathrm{P})$ & Flow Dependency & $\mathrm{F} 26_{\mathrm{t}}=1.82 * \mathrm{~F} 17_{\mathrm{t}}$ \\
\hline Bark storage $(\mathrm{P})$ & Storage Equation ${ }^{\mathrm{a}}$ & $\begin{array}{l}1.0 * 700 * \mathrm{~F} 29_{\mathrm{t}}-700 / 1.0 * \mathrm{~F} 30_{\mathrm{t}}+1.0 * \mathrm{SE}_{(\mathrm{t}-1)}+\mathrm{SE}_{\mathrm{t}}=0 \\
\mathrm{SE}_{0}=320000 \\
\mathrm{SE}_{\mathrm{t}}<=320000 \\
\mathrm{SE}_{\mathrm{T}}=320000 \\
1.0 * 700 * \mathrm{~F} 29_{\mathrm{t}}-700 / 1.0 * \mathrm{~F} 30_{\mathrm{t}}-700 / 1.0 * \mathrm{~F} 30_{(\mathrm{t}+1)^{-}} \\
700 / 1.0 * \mathrm{~F} 30_{(\mathrm{t}+2)}-700 / 1.0 * \mathrm{~F} 30_{(\mathrm{t}+3)^{-}}-700 / 1.0^{*} \mathrm{~F} 30_{(\mathrm{t}+4)^{-}} \\
700 / 1.0 * \mathrm{~F} 30_{(\mathrm{t}+5)}<=0\end{array}$ \\
\hline Incubation "Yoghurt" (D) & Batch Equation $^{\mathrm{b}}$ & $\begin{array}{l}1.0 * \mathrm{~F} 27_{\mathrm{t}}-1.0 * \mathrm{~F} 28_{(\mathrm{t}+5)}=0 \\
1.0 * \mathrm{~F} 27_{\mathrm{t}}<=1000000 * \mathrm{Y}_{\mathrm{t}} \\
\mathrm{Y}_{\mathrm{t}}+\mathrm{Y}_{(\mathrm{t}+1)}+\mathrm{Y}_{(\mathrm{t}+2)}+\mathrm{Y}_{(\mathrm{t}+3)}+\mathrm{Y}_{(\mathrm{t}+4)}<=1\end{array}$ \\
\hline Packaging machine 1 (D) & Boundary Top ${ }^{c}$ & $\begin{array}{l}\mathrm{F} 30_{0}-1.0 * \mathrm{~F} 30_{1}+1.0 * \mathrm{~F} 30_{2}+1.0 * \mathrm{~F} 30_{3}+1.0^{*} \mathrm{~F} 30_{4}+ \\
1.0 * \mathrm{~F} 30_{5}+1.0 * \mathrm{~F} 30_{6}+1.0 * \mathrm{~F} 30_{7}+1.0^{*} \mathrm{~F} 30_{8}+1.0 * \mathrm{~F} 30_{9} \\
+1.0 * \mathrm{~F} 30_{10}+1.0 * \mathrm{~F} 30_{11}+1.0 * \mathrm{~F} 30_{12}+1.0 * \mathrm{~F} 30_{13}+ \\
1.0 * \mathrm{~F} 30_{14}+1.0 * \mathrm{~F} 30_{15}+1.0 * \mathrm{~F} 30_{16}+1.0 * \mathrm{~F} 30_{17}+\end{array}$ \\
\hline
\end{tabular}




\begin{tabular}{lll}
\hline & & $1.0 * \mathrm{~F} 30_{18}+1.0 * \mathrm{~F} 30_{19}+1.0 * \mathrm{~F} 30_{20}+1.0 * \mathrm{~F} 30_{21}+$ \\
& & $1.0^{*} \mathrm{~F} 30_{22}+1.0 * \mathrm{~F} 30_{23}+1.0 * \mathrm{~F} 30_{24}=0$ \\
& $\mathrm{~F} 30_{0}>=15.5058$ \\
Packaging machine 1 (D) & Flow Equation & $\mathrm{F} 38_{\mathrm{t}}-5.5 * \mathrm{~F} 30_{\mathrm{t}}-5.5 * \mathrm{~F} 57_{\mathrm{t}}=0$ \\
Packaging machine 1 (D) & Flow Equation & $\mathrm{F} 57_{(\mathrm{t}<=8)}=0 ; \mathrm{F} 57_{(\mathrm{t}>8)}<=1,000,000$ \\
Packaging machine 1 (D) & Flow Equation & $\mathrm{F} 30_{(\mathrm{t}<=5)}=0 ; \mathrm{F} 30_{(\mathrm{t}>5)}<=1,000,000$ \\
Packaging machine 1 (D) & Flow Equation & $\mathrm{F} 30_{\mathrm{t}}+\mathrm{F} 57_{\mathrm{t}}<=6.06$ \\
\hline
\end{tabular}

${ }^{\mathrm{a}}$ The efficiencies of the storage in the pulp and paper mill equal 1.0 and the length of a time step is 700 hours.

b $1,000,000$ is a predetermined "large value" that is set within reMIND. 1.0 indicates the length of the time steps.

c Included in Cases D2-D6. The constraints are included for "Yoghurt". 1.0 indicates the length of the time steps. Similar constraints are included for "Sour cream" (F57). 
Table 10: Number of different functions in the cases studied. The intervals stated in the table are due to the different cases studied at the dairy and the pulp and paper mill, respectively.

\begin{tabular}{lcc}
\hline Function & Dairy $^{\mathrm{a}}$ & Pulp and paper mill $^{\mathrm{a}}$ \\
\hline Flow Dependency & $28-30$ & $18-20$ \\
Flow Equation & $7-8$ & 0 \\
Flow Relation & 4 & 2 \\
Boundary & 5 & $7-9$ \\
BoundaryTop & $0-2$ & 0 \\
Storage Equation & 8 & $0-2$ \\
Batch Equation & 2 & 0 \\
Logical Equation $_{\text {Investment Cost }}^{\mathrm{b}}$ & 0 & 0 \\
Source $^{\mathrm{c}}$ & $1-3$ & 0 \\
Destination $^{\mathrm{d}}$ & $4-6$ & 7 \\
Function Editor $^{\text {Total }}$ & $18-21$ & $2-3$ \\
\hline
\end{tabular}

\footnotetext{
${ }^{\mathrm{a}}$ The intervals are due to differences within the models for Cases D1-D6 and Cases P1-P4.

${ }^{\mathrm{b}}$ The different functions may be used for different purposes. In this case the Investment Cost functions are included to model the variable power demand fee.

${ }^{\mathrm{c}}$ The Source function is not described in the text. It is a function where a resource starts and a price may be entered for the resource, which is included as the coefficient in the objective function, for the flows that have been assigned the specific resource.

${ }^{\mathrm{d}}$ The destination function is not described in the text. It is a function where a resource ends. No input data is possible to enter.
} 
Table 11: Information about the size and solution times for the case studies. The intervals stated in the table are due to the different cases studied at the dairy and the pulp and paper mill, respectively.

\begin{tabular}{lcc}
\hline & Dairy $^{\mathrm{a}}$ & Pulp and paper mill $^{\mathrm{a}}$ \\
\hline Nodes & $34-38$ & $19-23$ \\
Branches & $56-60$ & $27-35$ \\
Total amount of variables & $3771-4151$ & 1014 \\
Binary integers & $62-68$ & 20 \\
Constraints & $5208-5688$ & $1554-1896$ \\
Model creation time $^{\mathrm{b}, \mathrm{c}}$ (CPU) & $10-12 \mathrm{sec}$. & $<2 \mathrm{sec}$. \\
Solution time $^{\mathrm{c}, \mathrm{d}}$ & $<2 \mathrm{sec}$. & $<1 \mathrm{sec}$.
\end{tabular}

${ }^{\mathrm{a}}$ The intervals are due to different sizes for the models for Cases D1-D6 and Cases P1-P4.

${ }^{\mathrm{b}}$ The time to create the MPS file.

${ }^{c}$ Using a computer with the following characteristics: AMD Turion, 992 MB of RAM.

${ }^{\mathrm{d}}$ Time for ILOG CPLEX 9.0 to solve the problem stated in the MPS-file. 
Table 12. System cost and peak demand at the dairy

\begin{tabular}{lccccccc}
\hline & D1 & D2 & D3 & D4 & D5 & D6 & Unit \\
\hline System cost & 138,900 & 129,800 & 126,200 & 126,100 & 124,000 & 123,900 & EUR/year \\
Peak (electricity & 1020 & 880 & 410 & 245 & 223 & 227 & $\mathrm{~kW}$ \\
$\begin{array}{l}\text { demand) } \\
\text { Peak (steam demand) }\end{array}$ & 1200 & 1010 & 782 & 914 & 823 & 981 & $\mathrm{~kW}$ \\
\hline
\end{tabular}


Table 13. System cost, oil and bark demand and electricity production at the pulp and paper mill

\begin{tabular}{lccccc}
\hline & P1 & P2 & P3 & P4 & Unit \\
\hline System cost & 76.4 & 75.7 & 74.2 & 73.1 & MEUR/year \\
Oil demand & 625 & 625 & 805 & 808 & GWh \\
Bark demand & 1022 & 1022 & 1062 & 1062 & GWh \\
Electricity production & 0 & 0 & 148 & 151 & GWh \\
\hline
\end{tabular}

